\title{
Las políticas de igualdad de género en tiempos de crisis. El caso gallego
}

\author{
Isabel DIZ OTERO \\ Universidad de Santiago de Compostela \\ isabel.diz@usc.es \\ Marta LOIS GONZÁLEZ \\ Universidad de Santiago de Compostela \\ martairene.lois@usc.es
}

Recibido: 10.10 .2014

Aceptado: 22.01.2015

\section{RESUMEN:}

El objetivo general de este artículo es analizar las reformas y transformaciones recientes de las políticas públicas de igualdad de género en Galicia a la luz del contexto de crisis económico financiera actual y el cambio de color político que se ha producido en los últimos años. Para lograr este objetivo general se intentan contestar las siguientes preguntas. ¿qué tipo de actuaciones en materia de igualdad se han llevado a cabo desde los gobiernos de la Xunta de Galicia? ¿Suponen el desarrollo de las políticas recientes algún cambio con respecto al grado de desarrollo de estas políticas antes del 2010? ¿Ha influido el color político en los cambios y la evolución de las políticas de género en Galicia?, ¿Es una cuestión de recursos? Finalmente, ¿se encuentra el mainstreaming institucionalizado?

Palabras clave: Bienestar; Igualdad; Políticas de género; Gasto social

\section{The gender equality policies in times of crisis. The Galician case}

\begin{abstract}
This article focus on the development and recent transformations of gender public policies in Galicia. The main aim is to test the influence of the current financial-economic crisis as well as the changes of political color of the party in government produced in the last years. In order to attain this general aim we are going to answer the following questions: Which type of equality political actions have been carried out by the governments of Xunta de Galicia? Has the development of these policies involved
\end{abstract}


some change since 2010? Has the development of this policies been influenced by the political color of the government? Do resources matter? And finally, is gender mainstreaming institutionalized?

Keywords: Welfare; Equality; Gender Policies; Social Cost

Entendemos que existe un Estado de bienestar cuando la mayoría de las actividades del Estado están dedicadas a promover el bienestar social. Las políticas sociales impactan en la vida de las personas y de la sociedad en general, son claves pues permiten universalizar los derechos sociales que se extienden a la ciudadanía. En la medida en que los derechos sociales se asumen como inviolables y la concesión de los mismos depende del desarrollo de una ciudadanía social y no de la actuación individual, podemos decir que las políticas sociales desmercantilizan el estatus de los individuos, los liberan de la consideración de mercancía pura, es entonces cuando las personas pueden sustentarse sin depender del mercado. Luego su bienestar ya no se supedita completamente a su participación en el mercado laboral, a la caridad o al apoyo familiar y, por lo tanto, estas políticas contribuyen, en mayor o menor medida, a corregir las fracturas sociales (Esping-Andersen, 1990:41).

Entre las políticas sociales se encuentran las políticas de igualdad de género dirigidas a eliminar las desigualdades existentes entre mujeres y hombres, son concebidas como un eje transversal en todas las políticas y han tenido un importante desarrollo durante las últimas décadas. Las políticas de género dan cuenta de la necesidad de erradicar las desigualdades de género en la estructura socioeconómica, política y cultural del sistema actual.

La crisis económico-financiera de estos últimos tiempos, sin embargo, ha traído consigo nuevas agendas políticas de recortes así como la redefinición del papel del Estado en la consecución del bienestar social y de la igualdad. Recientemente se han publicado numerosos artículos en relación a las consecuencias de esta crisis y a los cambios políticos producidos en el ámbito educativo, sanitario, laboral y cultural, pero son pocas las aproximaciones centradas de manera exclusiva en el campo de las políticas de igualdad de género. En ocasiones, se tiene la sensación de que los propios gobiernos las consideran políticas no prioritarias, políticas de segundo orden, a implementar mayormente en épocas de bonanza económica, como si la igualdad de género constituyera un valor prescindible, una suerte de lujo para los tiempos que corren. Ahora bien, al mismo tiempo, teniendo en cuenta que actualmente la ciudadanía en general siente aprecio por el Estado de Bienestar y desarrolla actitudes ciertamente positivas respeto a la igualdad entre mujeres y hombres, los gobiernos se inhiben de presentar abiertamente este argumento diseñando estrategias más sutiles destinadas a esquivar 
la posible resistencia ciudadana frente a los ajustes en las políticas sociales como la igualdad.

En este artículo nos gustaría acercarnos a este debate acerca de los cambios acontecidos en las políticas sociales y el bienestar en el contexto de crisis económica a través del análisis y descripción de las políticas de igualdad de género en Galicia. Estas se han ido aplicando en la Comunidad autónoma gallega desde los años noventa en un proceso creciente de transformación. Para conocer en profundidad su alcance y desarrollo abordaremos cuáles han sido hasta el momento las actuaciones en materia de igualdad que se han llevado a cabo con el objeto de evaluar qué cambios intraorganizativos y nuevas instituciones ha supuesto la incorporación de la perspectiva de género en el gobierno gallego. A partir de este análisis, se buscará responder a las preguntas de si se puede hablar de madurez y consolidación institucional de las políticas de género en Galicia y cuáles son las variables que afectan a este proceso. En este sentido, tanto la irrupción de la crisis económica como la presencia en el gobierno autonómico gallego del Partido Popular durante las dos últimas legislaturas obligan a una reconsideración de la evolución de estas políticas así como a una explicación de la influencia jugada por estas dos variables: la crisis y el color político del gobierno. En primer término, se trataría de valorar si una reducción de los recursos institucionales gallegos actuales para la igualdad, imputados al contexto de austeridad económica y racionalidad administrativa, constituyen una amenaza de futuro, y por lo tanto un peligro de desinstitucionalización de la igualdad de género. En segundo término, una vez constatado el avance que estas políticas experimentaron en el gobierno bipartito (Diz y Lois, 2011), se trata de considerar en qué medida está influyendo el color político del gobierno conservador del PP en la dirección, contenidos y posibles retrocesos de las políticas actuales de género.

El artículo se estructurará de la siguiente manera: en el primer apartado se aborda brevemente el marco de análisis utilizado para trazar la evolución y desarrollo de las políticas de igualdad de género en Galicia. A partir de este marco de análisis y la distinción que se hace en el mismo de las distintas etapas de aplicación de la estrategia del mainstreaming, se procede, en un segundo apartado, al análisis de cómo se han ido institucionalizando este tipo de políticas en Galicia atendiendo principalmente al color político del gobierno de la Xunta. En el tercer apartado se analizará el gasto social vinculándolo a la agenda en igualdad. Por fin, en las conclusiones se sintetizan las respuestas alcanzadas a las preguntas de partida de esta investigación. 


\section{EL MAINSTREAMING DE GÉNERO. UN MARCO PARA ANALIZAR LAS POLÍTICAS DE IGUALDAD EN CUATRO ETAPAS}

El desarrollo de las políticas de género en la Comunidad autónoma de Galicia, aunque un poco más tardío, fue paralelo a los avances en diferentes instituciones europeas y españolas. A partir de los años 90 se llevaron a cabo diversas actuaciones públicas en materia de igualdad entre mujeres y hombres, actuaciones que progresaron siguiendo en gran medida la lógica evolutiva de las distintas políticas de género, desde las políticas de igualdad de oportunidades hasta el mainstreaming (Diz y Lois,2011; Alonso, 2007). Esta última estrategia se incorpora por primera vez en el III Plan para la Igualdad de oportunidades de las mujeres gallegas, 1998-2001, aunque es con la Ley Gallega 7/2004, del 16 de julio, para la Igualdad de Mujeres y Hombres en la Comunidad Autónoma de Galicia que se afianza. Con esta ley, por un lado, queda introducido este principio del mainstreaming como mandato en la elaboración, en la ejecución y en el seguimiento de todas las políticas y de todas las actuaciones de la Xunta de Galicia $\mathrm{y}$, además, por otro lado, se incorporan algunos de los instrumentos necesarios para su implementación.

El objetivo fundamental de la estrategia del mainstreaming de género se centra en revisar todas las políticas públicas de modo que se elimine su sesgo de género y puedan contribuir activamente a la consecución de la igualdad entre mujeres y hombres. Desde su adopción por la mayoría de las administraciones han sido muchas las investigaciones dentro del campo de estudio de las políticas públicas que han demandado el desarrollo de marcos analíticos que nos permitan examinar cómo se ha ido incorporando esta compleja estrategia y cuál ha sido el resultado de la misma. Una propuesta, surgida en nuestro contexto académico más cercano, que supone un avance importante en este sentido es la de Alba Alonso (Alonso, 2014). Esta autora, siguiendo la propuesta de James Anderson (2003), identifica cuatro fases diferenciadas de esta estrategia: mandato, herramientas, resultado e impacto.

La autora subraya que, en primer lugar, el mainstreaming viene marcado por un cambio de enfoque. En este sentido, la simple enunciación de que es necesario ir más allá de las políticas tradicionales y revisar todas las actuaciones desde la perspectiva de género sería suficiente (mandato). Con el mandato se refiere a lo que Anderson denomina adopción (adoption), la cual apunta a la decisión- o no decisión- por una de las alternativas que se plantean para abordar un cierto problema. Para mejorar la viabilidad de esta estrategia resulta necesario dar un paso adelante y transcender la mera declaración de intenciones. Entraríamos en la fase de implementación en la que se precisaría de instrumentos concretos que hicieran más 
probable la aplicación de la política adoptada (herramientas). Con todo, podemos entender que el mainstreaming comienza cuando se logra constatar impactos concretos en las políticas habituales, esto es, cuando actuaciones sectoriales concretas se ven modificadas desde la perspectiva de género (outputs o resultado). Finalmente, la autora considera un umbral aún más ambicioso al entender que el mainstreaming solo se introduce cuando comienza a verse sus efectos en la sociedad (outcomes o impacto).

Como se ha dicho con anterioridad, en este artículo examinaremos cómo ha sido introducida la estrategia de mainstreaming en Galicia. Para ello analizaremos tres de los pasos identificados anteriormente: el mandato, las herramientas y, parcialmente, el resultado. Este análisis se hará haciendo especial referencia a tres etapas políticas diferenciadas, a saber, los inicios de la política de igualdad en Galicia que coinciden con el gobierno Fraga del año 89, la evolución de estas políticas durante la legislatura 2005-2009 de gobierno bipartito y, finalmente, las políticas llevadas a cabo por los gobiernos de Núñez Feijóo desde el año 2010.

Pues bien, La Ley 3/1991, del 14 de enero, de creación del Servicio Gallego de Promoción de la Igualdad del hombre y de la mujer (SGI) fue debatida y aprobada en el Pleno del Parlamento de Galicia el 18 de diciembre de 1990. Su aprobación supuso el punto de partida del feminismo institucional en la comunidad gallega al crear el primer organismo específico cuya finalidad principal era la de introducir las políticas de igualdad a nivel autonómico. Desde ese momento hasta el año 2005, el Partido Popular llevó a cabo políticas de igualdad de manera similar a como ocurrió en otras autonomías. En este sentido, a diferencia de otros contextos, estas políticas fueron paulatinamente aceptadas en España (Orbals, 2008; Threlfall, 1996), incluso en administraciones más conservadoras. En general, los partidos conservadores incrementaron sus actitudes favorables a las demandas feministas de igualdad de género como consecuencia de aspectos diversos como la necesidad de competir con la izquierda por el voto femenino, la creciente influencia de la legislación supranacional europea así como una mayor incorporación de las mujeres a la política (Ruiz Jiménez, 2002:239). No obstante, esta coincidencia con partidos situados en distinto lugar del espectro ideológico, no implica una coincidencia de resultado y contenido respecto a estas políticas. De acuerdo con Candice Ortbals, el color político del gobierno marcó una diferencia significativa entre los casos gallego y andaluz. En Galicia implicó un alejamiento de las demandas feministas y unas políticas públicas centradas en el papel de la mujer como madre y en el marco de la familia (Orbals, 2008).

A partir del cambio en el gobierno en el año 2005 y hasta principios del 2009 destacan dos aspectos importantes para el análisis de la evolución de las políticas de igualdad. Por una parte, el gobierno es ocupado por dos partidos de 
izquierda -PSdeG-PSOE y BNG- con una importante tradición de defensa de los intereses de las mujeres. Por otro lado, al tratarse de un gobierno bipartito, los dos partidos intentaron liderar la política de igualdad desde sus áreas de gobierno. Todo ello contribuyó a una mayor presencia de la misma en la agenda política.

En estos momentos, a finales de 2013, en el medio del proceso de crisis económico-financiera iniciado en 2008 y con una segunda legislatura del PP tras el gobierno bipartito, resulta imprescindible llevar a cabo una valoración del desarrollo de las actuaciones en materia de igualdad.

El análisis de estas tres etapas del desarrollo político nos permitirá además no dejar fuera del análisis las acciones específicas y las de igualdad de trato de manera que podamos contemplar las políticas de género en Galicia como un "taburete de tres patas" (Booth y Bennet, 2002) .

\section{LAS POLÍTICAS DE IGUALDAD DE GÉNERO EN ESPAÑA}

\subsection{La institucionalización de las políticas de género en Galicia. Un camino necesario para el desarrollo del mainstreaming.}

En todos los estudios que se plantean cuáles son los requisitos para una buena formulación de la estrategia del mainstreaming se incluye la necesidad de seguir realizando políticas específicas de igualdad. Además, como veremos, aunque esta estrategia supone una superación de las políticas de igualdad de trato y de las acciones específicas, es desde los organismos de igualdad y las distintas actuaciones de los mismos que se adopta el mainstreaming como política para luchar contra las desigualdades entre mujeres y hombres.

Como hemos dicho con anterioridad, y tal y como se refleja en la tabla 1, el Servicio Gallego de Igualdad (SGI) se constituyó desde 1990 como el organismo de igualdad $^{1}$ de la Xunta de Galicia. Su creación supuso el punto de partida del feminismo institucional en la comunidad gallega al crear el primer organismo específico cuya finalidad principal fue la de introducir las políticas de igualdad a nivel autonómico.

Entre las razones que llevaron al primer gobierno popular presidido por Fraga Iribarne a la creación del SGI, además de las señaladas en otras

\footnotetext{
${ }^{1}$ Los organismos de igualdad son definidos como entidades que forman parte de la estructura del gobierno y que tienen el propósito de promover la voz y los intereses de las mujeres (Krizsan et all, 2012)
} 
investigaciones como mimetismo institucional e influencia del contexto europeo (Alonso, 2007; Bustelo, 2004; Mahou y Bouzas, 2007), cobra un peso significativo la postura que en la oposición había mantenido este partido en la anterior legislatura. Durante el gobierno tripartito de la segunda legislatura, las iniciativas parlamentarias de demanda de creación de un organismo de igualdad autonómico fueron apoyadas por el grupo parlamentario popular en la oposición. Una vez llegado al poder, la marcha atrás se hacía difícil.

Tabla 1. Institucionalización de las políticas de igualdad en Galicia

\begin{tabular}{|c|c|c|c|}
\hline & $1990-2005$ & 2005-2009 & Después del 2009 \\
\hline $\begin{array}{l}\text { Organismos de } \\
\text { Igualdad }\end{array}$ & $\begin{array}{l}\text { Servicio Gallego } \\
\text { de Igualdad (91) }\end{array}$ & $\begin{array}{l}\text { Secretaría General de } \\
\text { Igualdad (05) }\end{array}$ & $\begin{array}{l}\text { Secretaría General } \\
\text { de Igualdad } \\
\text { Se suprime SGI } \\
\text { (Ley } 2 / 2010 \text { ) }\end{array}$ \\
\hline Planes de igualdad & $\begin{array}{l}\quad \text { I Plan para la } \\
\text { igualdad de } \\
\text { oportunidades de } \\
\text { las mujeres } \\
\text { gallegas, 1992- } \\
1994 \\
\qquad \text { II Plan para la } \\
\text { igualdad de } \\
\text { oportunidades de } \\
\text { las mujeres } \\
\text { gallegas, 1995- } \\
\text { 1997 } \\
\quad \text { III Plan para la }\end{array}$ & $\begin{array}{l}\text { V Plan del Gobierno } \\
\text { gallego para la igualdad } \\
\text { entre mujeres y hombres, } \\
\text { 2007-2010 }\end{array}$ & $\begin{array}{l}\quad \text { VI Plan } \\
\text { gallego para la } \\
\text { igualdad entre } \\
\text { mujeres y hombres. } \\
\text { Estrategia 2013- } \\
1015\end{array}$ \\
\hline
\end{tabular}




\begin{tabular}{|c|c|c|c|}
\hline & $\begin{array}{l}\text { igualdad de } \\
\text { oportunidades de } \\
\text { las mujeres } \\
\text { gallegas, 1998- } \\
2001 \\
\quad \text { IV Plan para la } \\
\text { igualdad de } \\
\text { oportunidades de } \\
\text { las mujeres } \\
\text { gallegas, 2002- } \\
2005\end{array}$ & & \\
\hline $\begin{array}{l}\text { Planes y } \\
\text { programas } \\
\text { sectoriales }\end{array}$ & & $\begin{array}{l}\text { El Plan de Fomento de la } \\
\text { Corresponsabilidad } 2007 \\
\text { Plan para la Salud } \\
\text { integral de las Mujeres } \\
2007 \\
\text { Programa Gallego de } \\
\text { Mujer y Ciencia 2008- } \\
2013\end{array}$ & \\
\hline $\begin{array}{l}\text { Normativa de } \\
\text { igualdad }\end{array}$ & $\begin{array}{l}\text { Ley Gallega } \\
\text { 7/2004, del } 16 \text { de } \\
\text { julio, para la } \\
\text { Igualdad de } \\
\text { Mujeres y Hombres }\end{array}$ & $\begin{array}{l}\text { Ley 2/2007, del } 28 \text { de } \\
\text { marzo, del trabajo en } \\
\text { igualdad de las mujeres } \\
\text { de Galicia } \\
\text { Ley 11/2007, del } 27 \text { de } \\
\text { julio, gallega para la } \\
\text { prevención y el }\end{array}$ & $\begin{array}{l}\text { Ley 5/2010, del } 23 \\
\text { de junio, por la que } \\
\text { se establece y } \\
\text { regula una red de } \\
\text { apoyo a la mujer } \\
\text { embarazada. }\end{array}$ \\
\hline
\end{tabular}




\begin{tabular}{|l|l|l|l|}
\hline & $\begin{array}{l}\text { tratamiento integral de la } \\
\text { violencia de género. }\end{array}$ & \\
& & & \\
\hline
\end{tabular}

Los trabajos más importantes que se han centrado en el estudio de los organismos de igualdad han señalado que la situación del organismo de igualdad en el organigrama del gobierno es importante para entender el papel que estos están llamados a desempeñar. Otras investigaciones centradas en el ámbito del Estado español sugieren utilizar, a la hora de explicar su fuerza institucional, el tipo de organismo del que se trata- referido a sí es un organismo autónomo, con presupuesto propio y creado por ley, o sí se trata, al contrario, de una dirección general, dependiente de un departamento del gobierno y de sus decisiones (Bustelo, 2004; Bustelo y Ortbals, 2007).

El diseño orgánico del SGI, así como otros organismos de igualdad autonómicos, siguen en su creación el modelo de la administración central: un organismo autónomo vinculado a un departamento percibido como de importancia secundaria, esto es, Trabajo y Servicios Sociales

Con todo, la consejería de adscripción del SGI ha ido cambiando a lo largo de los años de manera que experimenta una evolución positiva desde el punto de vista de la autoridad y de la capacidad del feminismo institucional en el nivel autonómico. La evolución cronológica de los órganos de adscripción ha sido la siguiente:

1. Consejería de Trabajo y Servicios sociales

2. Consejería de Familia, Mujer y Juventud

3. Consejería de promoción del Empleo, Mujer y Juventud

4. Consejería de Familia, Juventud, Deporte y Voluntariado Igualdad)

5. Vicepresidencia de la Igualdad y el Bienestar (Secretaría General de

6. Presidencia ( Secretaría General de igualdad) 
Esta evolución de la vinculación de las responsabilidades en igualdad de género y del SGI a los diferentes departamentos determina un distinto peso político y capacidad de influencia en el establecimiento de la agenda. Se puede identificar una división clara entre dos grandes períodos en lo referido al nivel organizativo. Un primero, antes del 2005, donde las políticas de género se sitúan en consejerías de bajo nivel de influencia y que actúan en ámbitos con poca incidencia para las restantes unidades de gobierno. Un segundo, posterior a 2005, en el que la estructura da un salto cualitativo. El Servicio Gallego de Igualdad se adscribe en este período al entorno de Presidencia. Este nivel, como se acaba de indicar, determina la presencia en un plano ventajoso y constituye además un nivel privilegiado para la introducción de la estrategia del mainstreaming, ya que favorece la capacidad de influencia y de coordinación con otros organismos para que incluyan la igualdad entre sus objetivos.

Desde este último punto de vista, esto es, su capacidad para favorecer la introducción del mainstreaming, también es preciso explicar la novedad que representó, en cuanto a diseño estructural de las políticas de igualdad, la adscripción de esta área de intervención a una Secretaría general incardinada en el gobierno, que como vemos en la tabla, se produce con el gobierno bipartito. Sin duda la capacidad ejecutiva de una secretaría general, frente a un organismo autónomo, significa situar en un primer plano de la agenda política la igualdad de género. Además la coexistencia de dos estructuras con responsabilidades en igualdad, garantizaba la necesidad de seguir con la estrategia dual reclamada aún como imprescindible para el avance de las políticas de igualdad.

Antes bien, esta situación ha mudado en las últimas legislaturas con la aprobación por parte del gobierno Feijoo de la Ley 7/2010, del 15 de octubre, por la que se suprime el organismo autónomo Servicio Gallego de Promoción de la Igualdad del hombre y de la mujer y se modifican determinados artículos de la Ley 2/2007, del 28 de marzo, del trabajo en igualdad de las mujeres de Galicia. Tras diecinueve años de funcionamiento, el SGI, que significó el punto de arranque del feminismo institucional en Galicia, fue suprimido aludiendo a criterios de racionalización de las estructuras. La ley argumenta que con la concentración de las funciones en la ya existente Secretaría General de Igualdad se gana en eficacia a la hora de aplicar el mainstreaming. Pues bien, a pesar de que podemos mantener la argumentación que hace la ley respecto a la estrategia del mainstreaming, distintos estudios y la evidencia empírica siguen mostrando que los organismos autónomos, como instituciones específicas de igualdad, son los más adecuados y extendidos en 
el conjunto de las autonomías ${ }^{2}$ para garantizar la continuidad y la estabilidad de las políticas de género al tiempo que impiden que estas sufran grandes retrocesos con los cambios de gobierno. Quizás nos encontramos en una de las situaciones señaladas por Alonso (Alonso, 2012) en las que el resultado de la incorporación del mainstreaming, en lugar de suponer un refuerzo para las políticas previas, en cierta medida ha puesto en peligro su supervivencia eliminando acciones específicas consolidadas y organismos de igualdad.

\section{Acciones}

El impulso de las políticas públicas de igualdad de género, esto es, de las medidas destinadas a eliminar las discriminaciones contra las mujeres, se concretó fundamentalmente a través de los planes de igualdad de oportunidades. Los planes de igualdad, constituyen un conjunto de medidas aprobadas por un gobierno para ser aplicadas por los departamentos que lo componen en un determinado intervalo temporal (Arranz, 2000:39). Son el principal instrumento de actuación de los organismos que trabajan para la eliminación de las relaciones desiguales de género. Desde los años 90, tal y como se observa en la tabla 1 , se han puesto en funcionamiento seis planes de igualdad:

I Plan para la igualdad de oportunidades de las mujeres, 1992-1994

II Plan para la igualdad de oportunidades de las mujeres, 1995-1997

III Plan para la igualdad de oportunidades de las mujeres 1998-2001

IV Plan para la igualdad de oportunidades de las mujeres, 2002-2005

$V$ Plan del Gobierno gallego para la igualdad entre mujeres y hombres, 2007-2010

VI Plan gallego para la igualdad entre mujeres y hombres. Estrategia $2013-1015$

El V Plan de igualdad supuso un salto cualitativo respecto de las políticas de género por dos motivos. En primer lugar, y por primera vez, nos encontramos delante de un Plan del Gobierno gallego. Los planes anteriores habían sido aprobados por el Pleno del Consejo del SGI, lo que significaba un compromiso menor por parte del gobierno del momento y una mayor dificultad para lograr que otros departamentos se sintieran forzados a la hora de cumplir las medidas recogidas en los mismos. En segundo lugar, la alusión explícita que se hace en el título a mujeres y hombres como destinatarios del V Plan, frente a la referencia sólo

\footnotetext{
${ }^{2}$ La organización exclusivamente política - direcciones generales en su mayoría- está presente tan sólo en los casos de Cantabria, Valencia, Madrid y Castilla-León.
} 
a las mujeres en los anteriores, evidencia el cambio de perspectiva a la hora de entender las políticas de igualdad como algo que beneficia a la sociedad en su conjunto y que necesariamente tiene que implicar también a los hombres. El VI Plan, vigente en la actualidad, ha mantenido esta nueva perspectiva, así como parte de la metodología de elaboración, contando con el conjunto de las consejerías del gobierno de la Xunta de Galicia.

Por otro lado, si se observan con detalle los contenidos de los planes, es posible identificar otro de los aspectos significativos de la evolución de las políticas de igualdad en Galicia, a saber, la adopción de la estrategia del mainstreaming.

Como se indica en el cuadro 2 referido a las áreas de actuación de los planes gallegos de igualdad, el mainstreaming se incorpora por primera vez en el III Plan de igualdad. Con todo, mientras que la mayor parte de los planes combinan políticas específicas con el nuevo mainstreaming, el V Plan avanza cualitativamente en esta estrategia diseñando líneas de actuación genéricas que persiguen el cambio de las condiciones estructurales que condicionan la persistencia de la desigualdad en las sociedades actuales ${ }^{3}$. El VI Plan, en el sentido subrayado anteriormente, también continúa con esta línea de actuación a partir de grandes ejes estratégicos que en este caso son los siguientes: avance de la gobernanza a favor de la igualdad, cambio de valores y modelos para la igualdad, aprovechamiento del talento femenino, conciliación corresponsable y calidad de vida, participación activa de las mujeres en la sociedad y acción integral para combatir la violencia de género. Ahora bien, en este último Plan en contraste con el anterior, se omiten las referencias al feminismo y se intensifican las medidas relacionadas en mayor medida con el empleo, la conciliación y la violencia de género.

Cuadro 2. Tipología de áreas de actuación en los planes gallegos de igualdad

\begin{tabular}{llllllll}
\hline Áreas de actuación & I & II & III & IV & V & VI \\
\hline Participación sociopolítica & & & & & & & \\
\hline
\end{tabular}

${ }^{3}$ Las líneas estratégicas de este $\mathrm{V}$ plan fueron cuatro: participación y empoderamiento; acceso a información y a los recursos; formación en igualdad entre mujeres y hombres; y tratamiento integral de la violencia de género. 
Sensibilización

Medios de Comunicación

Educación

Formación y empleo

Conciliación

Cultura

Deporte

Desarrollo sostenible

Salud

Servicios sociales

Violencia de género

mainstreaming

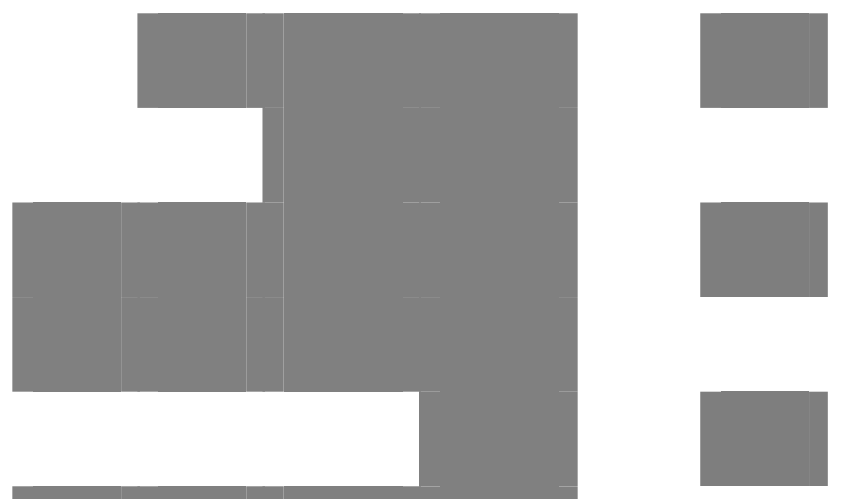

Fuente: elaboración propia a partir de Alonso (2007)

Junto con estos planes de igualdad de contenido general nos encontramos con tres planes específicos que podemos enmarcar en la estrategia del mainstreaming: El Plan de Fomento de la Corresponsabilidad y el Plan para la Salud integral de las Mujeres, aprobados los dos en el 2007 y el Programa Gallego de Mujer y Ciencia, aprobado en el año 2008. A diferencia del continuismo que observamos en los planes generales de igualdad, el gobierno actual no ha seguido este tipo de estrategia como forma de actuación. Desde que en el año 2009 la administración gallega es dirigida por el Partido Popular, no se han aprobado planes para el logro de la igualdad en ámbitos específicos de intervención política.

Otro tipo de estrategia más tardía en su aparición pero de gran relevancia en materia de igualdad la constituyen la aprobación de distintas leyes autonómicas. Nos referimos en concreto a la: 
Ley Gallega 7/2004, del 16 de julio, para la Igualdad de Mujeres y Hombres.

Ley 2/2007, del 28 de marzo, del trabajo en igualdad de las mujeres de Galicia.

Ley 11/2007, del 27 de julio, gallega para la prevención y el tratamiento integral de la violencia de género.

Ley 5/2010, del 23 de junio, por la que se establece y regula una red de apoyo a la mujer embarazada.

La aprobación de medidas legislativas supone un tipo de intervención que apuesta por herramientas de actuación más incisivas y más comprensivas de lo que resultan los planes (Aránzazu, 2003:67, Bustelo y Ortbals, 2007:211). Y es que una de las limitaciones de los planes de igualdad radica en el hecho de que las políticas tienden a permanecer exclusivamente en el ámbito del ejecutivo. Por el contrario, las leyes permiten que la acción en género se amplíe al ámbito legislativo y judicial. Con todo, resulta importante señalar, que las leyes autonómicas de igualdad siguen siendo consideradas como más próximas a una declaración de intenciones que a una norma de obligado cumplimiento.

\subsection{El mainstreaming de género en Galicia}

Como hemos señalado en la introducción, este apartado centrará su atención en revisar la incorporación y el desarrollo del mainstreaming en Galicia. En primer lugar se llevará a cabo un análisis de en qué momento y cuáles han sido los instrumentos que ha utilizado la administración gallega para introducir este nuevo enfoque para abordar la igualdad entre mujeres y hombres. En segundo lugar, se tratará el mainstreaming como herramienta, de modo que se realizará una revisión de los mecanismos que han sido desarrollados hasta la fecha para promover la revisión de todas las políticas desde la perspectiva de género. Por último, en tercer lugar, se analizará parcialmente cuál ha sido el resultado del mainstreaming. A través del la revisión de las leyes aprobadas por la Administración gallega en los últimos años se examinará en qué medida estas han introducido la perspectiva de género.

\section{Mandato}


Como se ha señalado anteriormente, la Administración gallega, utilizó los Planes de igualdad para la adopción de la estrategia del mainstreaming. Como se indicaba en el cuadro 2, el mainstreaming se incorpora por primera vez en el III Plan de Igualdad y a partir de ahí se ha ido profundizando esta estrategia pasando de una combinación de acciones sectoriales y transversales a planes donde la transversalidad es la nota común.

La adopción institucional del mainstreaming se produce, con todo, con la aprobación de una norma específica en materia de igualdad: La Ley Gallega 7/2004, del 16 de julio, para la Igualdad de Mujeres y Hombres. Esta ley establece el principio del mainstreaming en la elaboración, en la ejecución y en el seguimiento de todas las políticas y de todas las actuaciones de la Xunta de Galicia. Junto con esta disposición general, la ley, como veremos, incorpora a la actuación de la administración autonómica alguna de las herramientas del mainstreaming. Con la aprobación de la Ley Gallega 7/2004, Galicia se convierte en una de las comunidades pioneras en adoptar institucionalmente el mainstreaming. En cualquier caso, pese a los instrumentos que regula, esta ley no consigue la profundización necesaria para una buena implementación del mismo y está muy lejos de alcanzar el desarrollo legal de la Ley 4/2005, para la igualdad de mujeres y hombres aprobada por el país Vasco que se ha convertido en el referente para las normativas de igualdad españolas, incluida la principal ley a nivel estatal (Alonso, 2012).

En el año 2007 la actuación legislativa en materia de igualdad da un paso significativo en la incorporación del mainstreaming a la acción de la Administración autonómica. Este avance obedece a la incorporación del objetivo de la igualdad como dimensión central en la actuación de políticas sectoriales: violencia y trabajo. Así, la Ley 2/2007, del 28 de marzo, del trabajo en igualdad de las mujeres de Galicia, en su artículo primero, regula que su objeto es la integración de la dimensión de la igualdad de oportunidades entre mujeres y hombres en las competencias autonómicas sobre empleo y relaciones laborales. De la misma manera, la Ley 11/2007, del 27 de julio, gallega para la prevención y el tratamiento integral de la violencia de género establece como objetivo la adopción de medidas integrales dirigidas a la prevención y atención del tratamiento de la violencia ejercida contra las mujeres, así como la asistencia a las víctimas. Esta ley pretende dotar a los poderes públicos y a la sociedad gallega de un instrumento útil para prevenir, tratar y erradicar la violencia de género y, en fin, conseguir una verdadera realización del principio de igualdad.

Pero además, estas dos leyes han sido aprovechadas por el legislador gallego para profundizar el mainstreaming a través de la creación de muchas de las herramientas necesarias para su implementación. 
La última ley aprobada es la Ley 5/2010, del 23 de junio, por la que se establece y regula una red de apoyo a la mujer embarazada. En esta norma se ponen de manifiesto una nueva orientación en la dirección de las denominadas acciones "pro vida" que hacen pensar en un claro cambio de agenda de la administración popular respeto de las políticas de género del gobierno bipartito. En realidad, es una ley que guarda una relación muy estrecha con otras leyes autonómicas aprobadas por gobiernos del partido Popular ${ }^{4}$.

Esta nueva ley tiene como uno de sus objetivos garantizar el derecho a la vida en formación desde la concepción (Art. 1). Se trata, por lo tanto, de una propuesta de actuación en clara sintonía con algunas claves de las políticas en contra del aborto manifiestamente opuestas a las demandas feministas. De hecho, como elemento nuevo, considera a las mujeres embarazadas cómo unidad familiar de pleno derecho (art. 6.2).

\section{Herramientas}

Los mecanismos que han sido desarrollados hasta la fecha para promover la revisión de todas las políticas desde la perspectiva de género han sido de diverso tipo (Tabla 2): instrumentos de análisis, organizativos y de participación.

Con instrumentos de análisis nos referimos a las iniciativas que se ponen en marcha con el doble objetivo de mejorar el conocimiento sobre las desigualdades y contribuir a la revisión de las políticas públicas.

Como se ha mencionado más arriba, la Ley gallega de igualdad crea y obliga a la utilización de algunos de los instrumentos para la implementación de la estrategia que estamos analizando.

En concreto esta norma introduce uno de los instrumentos de análisis fundamentales: los informes de impacto de género. Así en el capítulo II se introducen dos artículos, el séptimo y el octavo, referidos a los Informes de Impacto

\footnotetext{
${ }^{4}$ Son la Ley 6/2009, de 30 de junio, de la Generalitat, de Protección a la Maternidad y la Ley 14/2008, de 18 de diciembre, por la que se crea y regula una red de apoyo a la mujer embarazada. Al parecer, el origen de estas leyes se encuentra en la iniciativa legislativa popular de la Fundación Red Madre. Es una red de asociaciones de asesoramiento las embarazadas impulsada en 2007 por el Foro Español de la Familia.
} 
de Género en la elaboración de las leyes y de las legislaciones elaboradas por la Xunta de Galicia. Los informes de impacto se establecen de manera obligatoria, aunque no vinculante, para proyectos y proposiciones de ley.

Muy vinculadas al diagnóstico de la realidad con perspectiva de género imprescindibles en la elaboración de los informes se incorpora a la ley otro de los instrumentos de análisis relevantes. Así, la disposición adicional primera fija, con la finalidad de facilitar la aplicación del principio de mainstreaming, que las estadísticas e investigaciones con eventual repercusión en cuestiones de género, realizadas por la comunidad autónoma se encuentren desagregadas por sexos. Este mandato se va a concretar posteriormente en la Leyes del Plan Gallego de estadística para los periodos 2007-2011 y 2012-2016.

Por último, hay que hacer referencia a la formación en género para el personal de las administraciones públicas. Hasta el 2011 esta formación había sido introducida de manera poco sistemática, principalmente dirigida a personal que trabaja en el área de igualdad y casi siempre con carácter voluntario. A partir de este año se crea la Escuela gallega de Profesionales de la igualdad con la finalidad de responder a esta necesidad de formación en género del personal de las administraciones públicas. Sin embargo, analizando hasta la fecha los programas ofertados por esta escuela, parece que no han sido superadas las características anteriores. La formación sigue siendo voluntaria y dirigida a personal de la administración que trabaja en el ámbito de la igualdad.

Se hacía referencia más arriba a que las leyes en materia de género aprobadas por el bipartito habían avanzado de manera importante en la implementación del mainstreaming. Se puede decir que esta normativa estableció los instrumentos organizativos y de participación con los que contamos en la actualidad.

Con respecto a los primeros en este periodo se crean dos estructuras especializadas de área: el Servicio de igualdad laboral y la Unidad Mujer y Ciencia. El Servicio de igualdad laboral se regula en la Ley 2/2007, del 28 de marzo, del trabajo en igualdad de las mujeres de Galicia. Se trata de la unidad administrativa de igualdad del departamento de la Administración autonómica competente en materia de trabajo, que funcionará coordinada y vinculada funcionalmente con el departamento de la administración autonómica competente en materia de igualdad. Las funciones que se le atribuyen son de asesoramiento en materias de género, impulso de la participación equilibrada de mujeres y hombres en los puestos de trabajo del citado departamento, diseño de formación en materia de igualdad, colaboración con el departamento competente en materia de igualdad en la elaboración de los informes de impacto de género de las normas del 
departamento de la administración autonómica competentes en materia de trabajo, recepción de las estadísticas que este elabora, promoción del principio de igualdad, asesoramiento a empresas, revisión de convenios colectivos, elaboración de dictámenes y vigilancia sobre el cumplimiento de esta ley

La Unidad de Mujer y Ciencia se instituye a través del Decreto 33/2007 del 1 de marzo. Se trata de un organismo especializado en potenciar la presencia de las mujeres en los ámbitos científico y tecnológico y que cuenta con la participación de tres departamentos gubernamentales: la Secretaría General de Igualdad, la Consejería de Educación y la Consejería de Innovación e Industria. La colaboración de estas dos últimas representa un claro impulso para la aplicación del mainstreaming, ya que son departamentos que entran a formar parte del feminismo institucional, mostrando un claro compromiso con la eliminación de las desigualdades y aportando a la vez su experticia (Alonso, 2008a; 2008b).

Con todo, el balance que hay que hacer del grado de implementación de estas estructuras en la actualidad es negativo. Con la Ley 7/2007 aprobada en octubre, la Unidad administrativa de Igualdad del departamento de trabajo deja de estar vinculada a la Secretaría General de Trabajo, para coordinarse sólo con el departamento de la administración autonómica competente en materia de igualdad. Pierde así, en cierta manera, mucho de su carácter transversal y capacidad de incidencia, al no ser el propio departamento de trabajo el que dirige la política de igualdad en su ámbito. Por su parte, no es tan negativa la evaluación de la Unidad Mujer y Ciencia. Si podemos decir que su actividad ha perdido mucho del dinamismo que la caracterizó los primero años. Además la implicación de los departamentos de Educación e Innovación e industria, tan estratégica en los primeros momentos se ha debilitado de manera significativa.

Un segundo grupo de herramientas organizativas que nacieron durante el gobierno bipartito corresponde a estructuras centradas en mejorar la coordinación entre departamentos, o entre niveles institucionales, con el fin de facilitar la revisión de todas las políticas. Se crea de esta manera un organismo de coordinación entre consejerías: Comisión Interdepartamental de Igualdad. La creación de esta comisión, que ya funcionó para la elaboración del V Plan del Gobierno gallego de igualdad entre mujeres y hombres de Galicia, se regula en la Ley 11/2007, del 27 de julio, gallega para la prevención y el tratamiento integral de la violencia de género como un órgano colegiado e institucional de la Xunta.

Así como la Comisión interdepartamental de Igualdad fue fundamental para la elaboración del V Plan del año 2007, no ha actuado de la misma manera en la elaboración del VI Plan, en el que la participación de los distintos departamentos del ejecutivo se realizó a través de la Comisión de Secretarías Generales Técnicas. 
Esta última experiencia parece ser la inspiradora de lo regulado en el Decreto $157 / 2012^{5}$, en el que se desarrolla esta Comisión. Esta Comisión estará integrada por las Secretarías Generales Técnicas en calidad de vocales. La presidirá la persona titular del departamento al que se adscriba la política de igualdad y la vicepresidencia será ocupada por la persona responsable de esta política.

Por último, entre las estructuras creadas por el bipartito hay que mencionar el Observatorio gallego de la violencia de género, que también se ha desarrollado a través del Decreto 157/2012. Se trata de un tipo de estructuras cuyo objetivo fundamental es crear conocimiento sobre algún aspecto de la realidad de las mujeres y analizar y evaluar su situación.

La participación ciudadana en el proceso de elaboración de las políticas públicas de igualdad se garantiza con la creación de dos organismos: el Consejo gallego de las mujeres y Consejo gallego de participación de las mujeres en el ámbito del empleo y de las relaciones laborales. La Ley 11/2007, del 27 de julio, gallega para la prevención y el tratamiento integral de la violencia de género crea el Consejo Gallego de las Mujeres como órgano colegiado e institucional de Galicia, de carácter consultivo, de participación y asesoramiento en materia de políticas de igualdad. Además, la Ley 2/2007, del 28 de marzo, del trabajo en igualdad de las mujeres de Galicia también regula el Consejo Gallego de participación de las mujeres en el ámbito de empleo y de las relaciones laborales como órgano de participación mediante un canal de libre adhesión de las asociaciones de mujeres, donde se integren las secretarías de mujeres de los sindicatos y de la confederación de empresarios y empresarias de Galicia y con representación del departamento de la administración autonómica en materia de igualdad. Posteriormente el Decreto 157/2012 declara extinguido organizativamente este último y asigna sus funciones al Consejo Gallego de las mujeres. Este mismo decreto regula la formación y composición del mismo. Este Consejo está constituido desde principios de marzo del 2014 después de un proceso de selección en el que participó parte del tejido asociativo gallego de mujeres.

${ }^{5}$ Decreto 157/2012, del 5 de julio de 2012, por el que se desarrolla la Ley 11/2007, del 27 de julio, gallega para la prevención integral de la violencia de Género en lo relativo a los órganos consultivos y de participación. 
Tabla 2. Las herramientas del Mainstreaming de género en Galicia

\begin{tabular}{|c|c|c|c|}
\hline Herramientas: & $1990-2005$ & $2005-2009$ & Después del 2009 \\
\hline \multicolumn{4}{|l|}{ instrumentos de análisis } \\
\hline Informes de impacto & $\begin{array}{l}\text { Ley Gallega } \\
4 / 2004\end{array}$ & & \\
\hline Formación en género & & & $\begin{array}{l}\text { Desde 2011: Escuela } \\
\text { gallega de } \\
\text { profesionales de } \\
\text { igualdad }\end{array}$ \\
\hline Estadísticas desagregadas & & $\begin{array}{l}\text { Ley del Plan Gallego de } \\
\text { Estadística 2007-2011 ( } \\
\text { se procurará) }\end{array}$ & $\begin{array}{l}\text { Ley del Plan gallego } \\
\text { de Estadística } \\
2012 / 2016 \\
\text { (obligatorio por } \\
\text { cumplimiento de la } \\
\text { ley)* }\end{array}$ \\
\hline \multicolumn{4}{|l|}{$\begin{array}{l}\text { instrumentos } \\
\text { organizativos }\end{array}$} \\
\hline Unidades de género & & $\begin{array}{l}\text { Unidad de Género en la } \\
\text { Consejería de trabajo } \\
\text { (creada en la ley } 2 / 2007 \text { ) } \\
\text { Unidad mujer y ciencia } \\
\text { (creada en Decreto } \\
33 / 2007 \text {, del } 1 \text { de marzo, } \\
\text { por el que se crea la } \\
\text { Unidad de Mujery } \\
\text { Ciencia de Galicia, }\end{array}$ & $\begin{array}{l}\text { Modificada en la ley } \\
2 / 2010\end{array}$ \\
\hline
\end{tabular}




\begin{tabular}{|c|c|c|}
\hline $\begin{array}{r}\text { Comisiones } \\
\text { Interdepartamentales }\end{array}$ & $\begin{array}{l}\text { Comisión } \\
\text { Interdepartamental de } \\
\text { Igualdad (Ley 11/2007, } \\
\text { del } 27 \text { de julio, gallega } \\
\text { para la prevención y el } \\
\text { tratamiento integral de la } \\
\text { violencia de género) }\end{array}$ & $\begin{array}{l}\text { Decreto 157/2012, } \\
\text { del } 5 \text { de julio de 2012, } \\
\text { por el que se } \\
\text { desarrolla la Ley } \\
\text { 11/2007, del } 27 \text { de } \\
\text { julio, gallega para la } \\
\text { prevención integral } \\
\text { de la violencia de } \\
\text { Género en lo relativo } \\
\text { a los órganos } \\
\text { consultivos y de } \\
\text { participación }\end{array}$ \\
\hline Organismos especializados & $\begin{array}{l}\text { Observatorio gallego de } \\
\text { la violencia de Género } \\
\text { creado en la Ley } 11 / 2007\end{array}$ & $\begin{array}{l}\text { Se desarrolla en } \\
\text { Decreto 157/2012, del } \\
5 \text { de julio de 2012, } \\
\text { por el que se } \\
\text { desarrolla la Ley } \\
\text { 11/2007, del } 27 \text { de } \\
\text { julio, gallega para la } \\
\text { prevención integral } \\
\text { de la violencia de } \\
\text { Género en lo relativo } \\
\text { a los órganos } \\
\text { consultivos y de } \\
\text { participación }\end{array}$ \\
\hline \multicolumn{3}{|l|}{$\begin{array}{l}\text { Herramientas: } \\
\text { instrumentos de } \\
\text { participación }\end{array}$} \\
\hline Consejos de mujeres & $\begin{array}{l}\text { Consejo Gallego de las } \\
\text { Mujeres creado por la }\end{array}$ & $\begin{array}{l}\text { Se desarrolla en } \\
\text { Decreto 157/2012, del }\end{array}$ \\
\hline
\end{tabular}




\begin{tabular}{|l|l|l|}
\hline & Ley 11/2007 & $\begin{array}{l}5 \text { de julio de 2012, } \\
\text { por el que se } \\
\text { desarrolla la Ley } \\
11 / 2007, \text { del } 27 \text { de } \\
\text { julio, gallega para la } \\
\text { prevención integral } \\
\text { de la violencia de } \\
\text { Género en lo relativo } \\
\text { a los órganos } \\
\text { consultivos y de } \\
\text { participación. }\end{array}$ \\
\hline
\end{tabular}

\section{Resultados}

Para terminar el análisis sobre el desarrollo de la estrategia del mainstreaming en Galicia se hará referencia a los resultados de este proceso. Nos referimos con ello y como se ha mencionado más arriba a la presencia de cambios concretos en las políticas sectoriales. Es decir, se trata de corroborar que las distintas políticas han sido revisadas para promover la igualdad de mujeres y hombres.

La metodología que vamos a utilizar en este artículo es la del análisis de las leyes aprobadas por el parlamento de Galicia. Somos conscientes de que la introducción de la perspectiva de género en la legislación es solo un elemento en el proceso de elaboración de una política pública y por lo tanto supone una corroboración parcial de que las políticas públicas están siendo revisadas para promover la igualdad. Con todo, creemos que este análisis puede arrojar algo de luz sobre la relevancia que está empezando a tener este proceso.

En la tabla 3 se resumen el porcentaje de leyes aprobadas en las tres últimas legislaturas que incorporan alguna medida relacionada con la promoción de la igualdad de género. Como se puede observar, en términos generales, ha sido abundante la normativa en la que se ha introducido algún tipo de mandato o acción para lograr una mayor igualdad. Así en la VII legislatura el porcentaje de leyes que introducen este tipo de medidas alcanza un $63,8 \%$ y aunque en las siguientes legislaturas esta tasa baja algún punto se puede mantener que el 51,8\% sigue siendo un porcentaje significativo. 
Tabla 4. Leyes que incluyen la perspectiva de género por legislatura

\begin{tabular}{|l|r|r|}
\hline & $\begin{array}{l}\text { VII } \\
\text { Legislatur } \\
\text { a }\end{array}$ & $\begin{array}{l}\text { VIII-IX } \\
\text { legislaturas }\end{array}$ \\
\hline Leyes aprobadas & 47 & 49 \\
\hline Leyes que introducen medidas en igualdad de género & 30 & 25 \\
& $63,8 \%$ & $51,8 \%$ \\
\hline
\end{tabular}

La tabla 5, que presenta un análisis más cualitativo sobre el tipo de medidas que se han introducido, no nos permite una valoración tan positiva. En efecto, de la tabla se desprende que la mayoría de la normativa y, por lo tanto, de las políticas sectoriales introducen la defensa de la igualdad como principio general de actuación. Sin embargo, han sido muy pocas las normativas que han introducido acciones concretas para luchar contra las desigualdades existentes. Además las otras áreas de actuación se limitan a la presencia paritaria en órganos de decisión, formación en género, presencia en profesiones y violencia de género, quedando fuera de la intervención pública aspectos trasformadores de los roles de género como la educación, la corresponsabilidad o la sexualidad. Por último, también se observa que en la octava y lo que llevamos de novena legislaturas, las medidas concretas tienen en mayor medida carácter voluntario y menor poder transformador.

Con todo, la metodología empleada impone precaución en las conclusiones y emplaza a la necesidad de la realización de investigaciones centradas en el análisis de políticas públicas sectoriales. 
Tabla 5. Medidas de igualdad de género en la leyes por legislatura

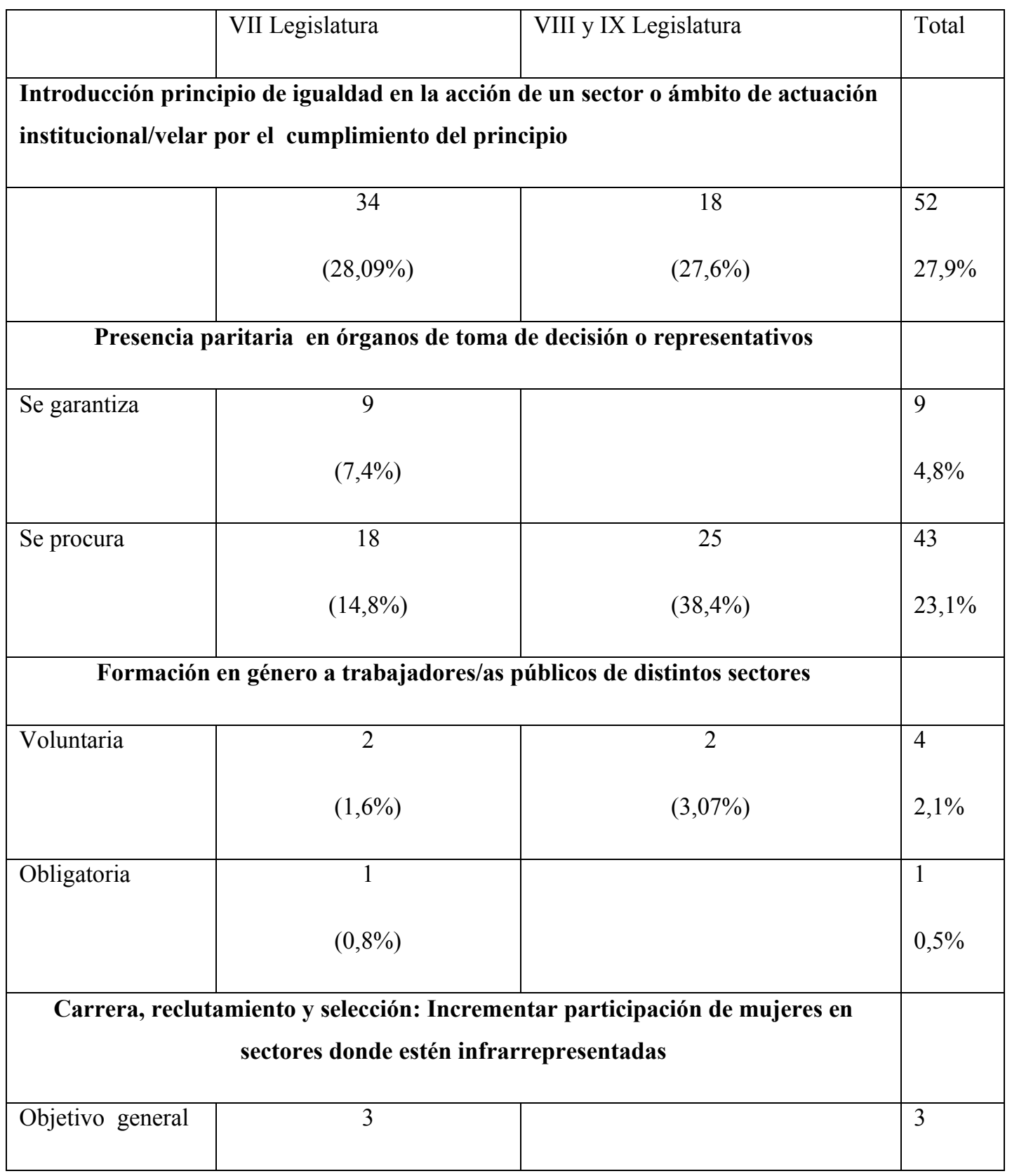




\begin{tabular}{|c|c|c|c|}
\hline & $(2,4 \%)$ & & $1,6 \%$ \\
\hline $\begin{array}{l}\text { Discriminación } \\
\text { positiva }\end{array}$ & $\begin{array}{c}3 \\
(2,4 \%)\end{array}$ & & $\begin{array}{l}3 \\
1,6 \%\end{array}$ \\
\hline Acción positiva & $\begin{array}{c}12 \\
(9,9 \%)\end{array}$ & $\begin{array}{c}10 \\
(15,3 \%)\end{array}$ & $\begin{array}{l}22 \\
11,8 \%\end{array}$ \\
\hline \multicolumn{4}{|c|}{ Medidas destinadas a las víctimas de violencia de género } \\
\hline $\begin{array}{l}\text { Atención/principi } \\
\text { o de actuación }\end{array}$ & $\begin{array}{c}6 \\
(4,9 \%)\end{array}$ & $\begin{array}{c}1 \\
(1,5 \%)\end{array}$ & $\begin{array}{l}7 \\
3,7 \%\end{array}$ \\
\hline Recursos víctimas & $\begin{array}{c}17 \\
(14,04 \%)\end{array}$ & $\begin{array}{c}2 \\
(3,07 \%)\end{array}$ & $\begin{array}{l}19 \\
10,21\end{array}$ \\
\hline Conciliación & $\begin{array}{c}6 \\
(4,9 \%)\end{array}$ & & $\begin{array}{l}6 \\
3,2 \%\end{array}$ \\
\hline Otras & $\begin{array}{c}10 \\
(8,2 \%)\end{array}$ & $\begin{array}{c}7 \\
(10,7 \%)\end{array}$ & $\begin{array}{l}17 \\
9,1 \%\end{array}$ \\
\hline TOTAL & 121 & 65 & 186 \\
\hline
\end{tabular}

En los últimos años se ha debatido bastante acerca de que aspectos relacionados con las políticas sociales, el bienestar y la igualdad de género son fundamentales para valorar el alcance de las reformas y los posibles retrocesos. Hasta el momento, con el fin de determinar el estado de desarrollo de la igualdad en Galicia en este artículo hemos analizado la evolución y los cambios experimentados en los últimos años respecto al mandato, las herramientas y los resultados sobre la normativa vigente. Ahora bien, uno de los indicadores más empleados para medir el 
cambio, la expansión o el retroceso de una política social sigue siendo el gasto social, es decir, los recursos económicos destinados por un gobierno a las políticas sociales. El mayor o menor porcentaje de gasto social, en general, permite evaluar el grado de compromiso político adquirido por los ejecutivos.

Cuando se aborda el tema del gasto social, sin embargo, es preciso ser especialmente cuidadoso. Recientemente este debate ha sido objeto de importantes críticas en la medida en que el empleo del denominado esfuerzo en bienestar - gasto social como porcentaje del PIB- no permite explicar siempre el alcance real que la reducción que determinadas políticas sociales tiene sobre las personas. De ahí, por tanto, la necesidad de analizar mejor cómo se distribuye y cuál es el contexto en el que se realiza el gasto social; por ejemplo, evaluando la duración y tipo de prestaciones, el tipo de programas, si tienen un carácter universal o restrictivo, si se producen recortes importantes en unos programas y expansión en otros y cuáles son, en definitiva, las consecuencias concretas que tienen para las personas y sus derechos sociales.

La limitada propensión de los gobiernos a reconocer recortes en los programas de bienestar está vinculada durante los últimos años de manera exclusiva a afianzar el discurso de la no reducción del gasto social. De manera que una vez anunciado por parte de un gobierno que el gasto social no se reduce de forma significativa sino que incluso se mantiene, se concluye que no se producen retrocesos ni recortes.

En general, pese a la declaración gubernamental de garantía de "blindaje del gasto social" durante la crisis, en la evolución funcional de los presupuestos gallegos relativos a Sanidad, Educación, Acción y Promoción social, y de acuerdo con los datos del Ministerio de Hacienda y Administraciones, se observa que a partir de 2011 se produce un descenso importante del gasto social. Esta caída es más acusada en el ejercicio 2014, donde baja más de 5 puntos porcentuales y situándose en los niveles más bajos de los últimos años (ver gráfico 1).

Grafico 1. Gasto social en Galicia 


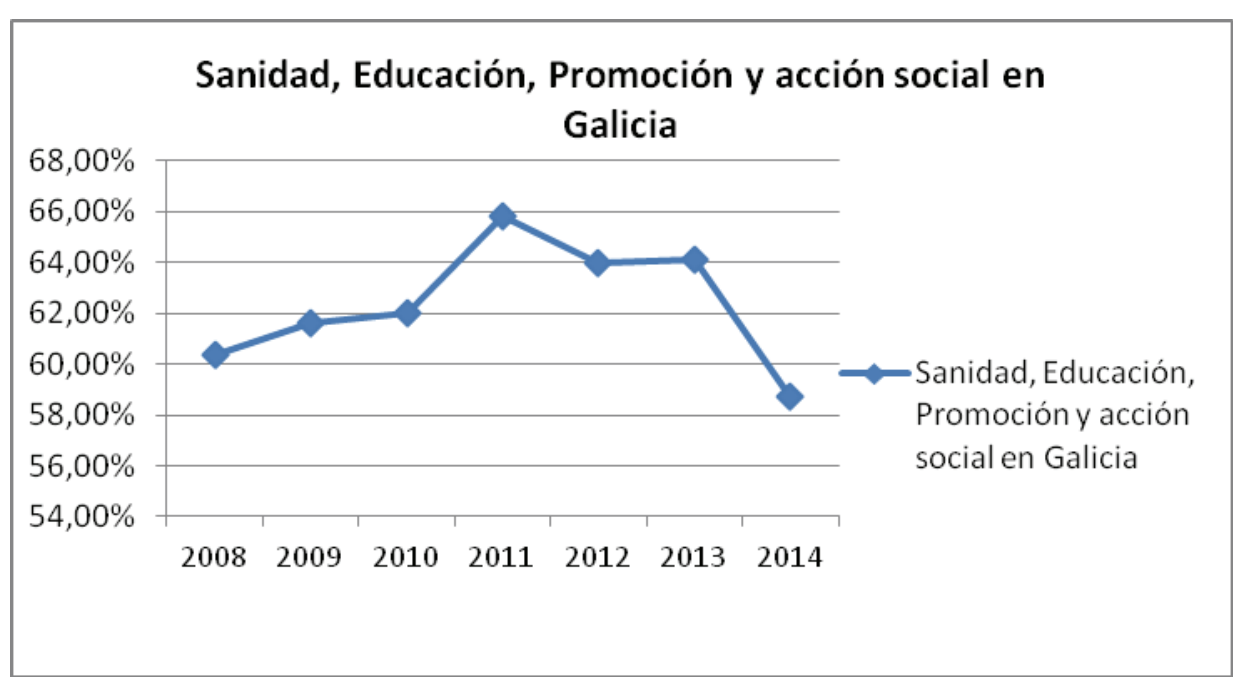

Fuente: elaboración propia a partir de datos Ministerio de Hacienda

Por su parte, si nos centramos en el gasto dirigido a las políticas de igualdad de género hay que tener en cuenta que las nuevas ingenierías de gasto social poseen contornos difusos que dificultan tanto el análisis como la interpretación de las mismas. En efecto, las políticas vinculadas a la igualdad de género comprenden un amplio espectro de actuaciones orientadas a corregir las situaciones de desigualdad entre mujeres y hombres y por lo tanto, en la medida en que obedecen a una estrategia dual de aplicación de políticas específicas de igualdad al tiempo que de aplicación transversal en todas las políticas - educación, sanidad, vivienda, empleo, dependencia, etc...- no resulta fácil medir el compromiso de gasto concreto así cómo estas políticas se ven afectadas por los recortes.

Ahora bien, si atendemos a los recursos presupuestarios destinados a la igualdad en los últimos años, observamos una sustantiva reducción del gasto en igualdad en los programas impulsados por la propia Secretaria General de Igualdad. Así, como vemos en el gráfico 2, respecto al gasto realizado en programas dedicados a las políticas de género - igualdad, conciliación y violencia de géneropara el período 2009-2014, se observa una caída pronunciada del gasto especialmente a partir de 2010. Los recortes afectan a los programas generales de igualdad y conciliación - reducción del $80 \%$ - manteniéndose prácticamente estable 
con una leve subida del $2 \%$ en las políticas contra la violencia de género ${ }^{6}$. Esta caída que se estabiliza en el tiempo da cuenta de la poca relevancia que la política específica de igualdad de género tiene en el marco de la actuación gubernamental durante el período que se identifica con las crisis económica. Además, y en comparación con los descensos en el gasto de las políticas sociales - sanidad, educación, acción y promoción social- se confirma que las políticas género poseen un peso residual dentro de las actuales políticas sociales.

Gráfico 2. Gasto en políticas de género (Miles de euros)

${ }^{6}$ El dato es resultado de evaluar los presupuestos dedicados a los programas de las políticas de género durante ese período. La fuente de datos empleada fueron los presupuestos anuales publicados en el DOGA y los informes de presupuestos de la Consejería de Hacienda. Los presupuestos incluían tres programas específicos, a saber, la promoción de las mujeres o de la igualdad entre mujeres y hombres, la conciliación y la lucha contra la violencia de género. 


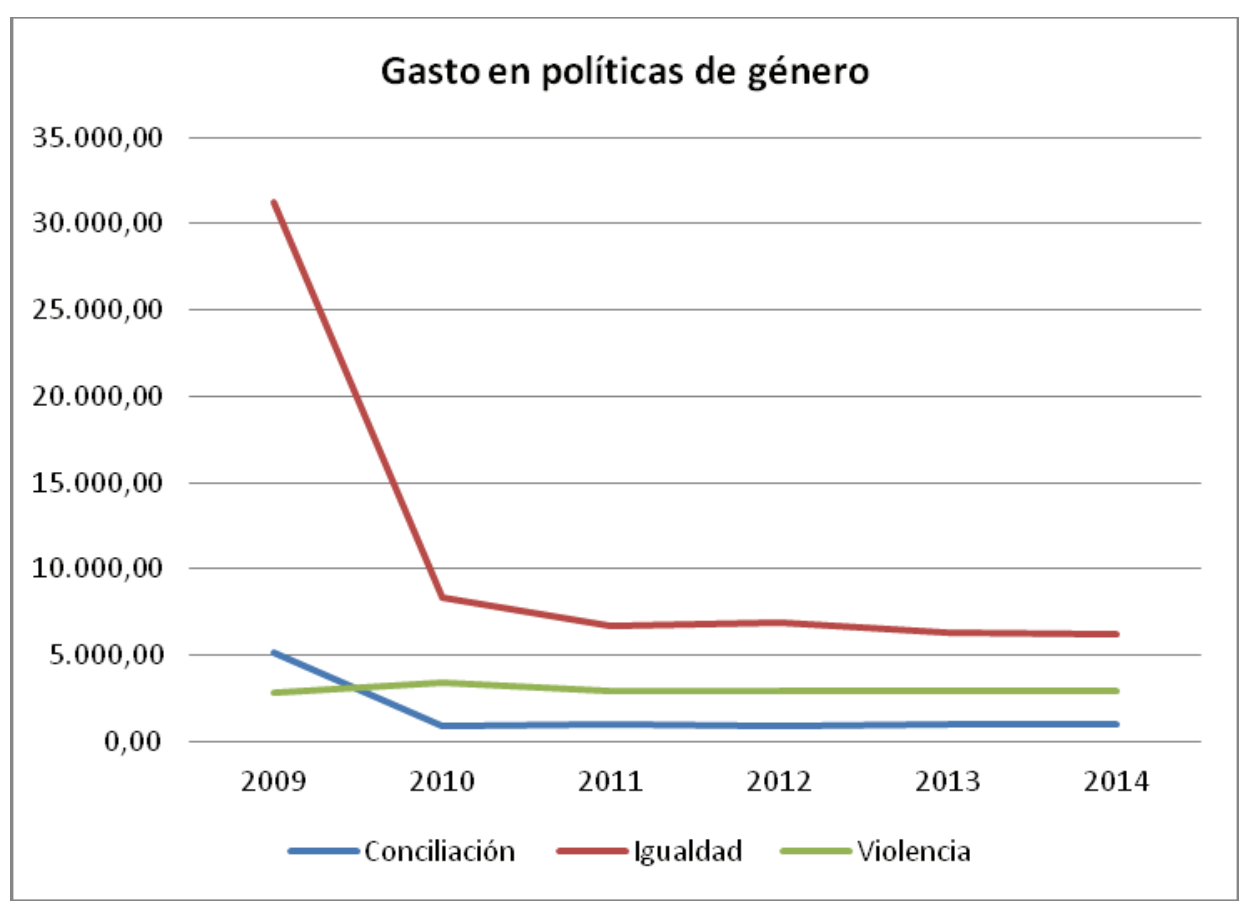

Fuente: Consejería de Hacienda. Presupuestos

Asimismo, esta reducción importante del gasto social en igualdad ha ido acompañada de un giro sustantivo en algunos de los contenidos de las políticas gallegas de género en las dos últimas legislaturas. Las actuaciones y los programas se han centrado mayormente en temas de conciliación, violencia, empleo, familia y apoyo a la maternidad, pasando a un segundo plano, o simplemente desapareciendo, los temas relativos a la salud sexual y reproductiva de las mujeres, las políticas científicas con perspectiva de género, las políticas dirigidas a la implicación de los hombres en la igualdad, la corresponsabilidad, etc.

La Ley 5/2010, del 23 de junio, por la que se establece y regula una red de apoyo a la mujer embarazada muestra un cambio de agenda política hacia un proyecto con vocación claramente no feminista. A partir de su aprobación la Secretaría General de Igualdad comenzó a subvencionar la "Red Madre" y su programa de fomento de la maternidad. Se trata de políticas que apuestan porque las mujeres gestantes continúen, en cualquier caso, adelante con sus embarazos, con independencia de que se encuentren o no en situación para ello. Entre las acciones positivas transversales diseñadas por el gobierno del PP se encuentran también las ayudas en materia de vivienda para las que computaría los fetos como miembros de 
la unidad familiar. Además el Plan de Apoyo a la Mujer Embarazada aprobado por Núñez Feijóo expresa con claridad la intención de apoyar embarazos no deseados mediante procedimientos de acogida y adopción de medidas como el denominado "protocolo de renuncia de bebés". Procedimientos claramente dirigidos a los grupos de mujeres en situación de vulnerabilidad, menores, sin recursos, inmigrantes, etc.-. Al mismo tiempo, observamos que a través de esta nueva agenda de la igualdad de género de apoyo a la maternidad, también expresada eufemísticamente como políticas de "dinamización geográfica" se hace hincapié en políticas que no comprenden al conjunto total de las mujeres. Además estas políticas restringen su autonomía para decidir sobre la maternidad. Son políticas focalizadas en el rol exclusivamente de las mujeres como madres que invisibilizan a los hombres como corresponsables en las tareas de cuidado.

\section{CONCLUSIONES}

En términos generales se puede concluir que el estado de madurez e institucionalización de las políticas de igualdad de género alcanzado en los últimos años y enmarcado en el denominado estado de bienestar ha comenzado a dar muestras de importantes retrocesos que están poniendo en peligro la propia institucionalización. La crisis económico-financiera y el color político del gobierno actual parecen estar detrás de este nuevo escenario.

Como vimos, en los años 90 se produjo un progreso continuo motivado, en gran medida, por la lógica evolutiva y la aparición de los distintos organismos, instrumentos y estrategias propias das políticas de igualdad de género. A partir de la creación del SGI, las políticas gallegas experimentaron una evolución constante. En primer término, se desarrollaron como instrumentos específicos de actuación los planes plurianuales de igualdad y normativa específica. La aprobación de los diferentes planes determinó una continua revisión y ampliación de la agenda política. Paralelamente, se produjeron modificaciones en la estructura organizativa con la que se ha abordado la igualdad. Con el fin de implementar el mainstreaming de género se pusieron en marcha herramientas organizativas - aquellas que permitieron iniciar la implementación de esta estrategia-, participativas -de inclusión das voces das mujeres- y analíticas - destinadas a la realización de estudios acerca de las desigualdades entre mujeres y hombres-.

Si atendemos al factor político, el período de legislatura iniciado en 2005 con el gobierno bipartito supuso un salto cualitativo tanto respeto al contenido de las políticas como al aumento de las estructuras. Los nuevos planes e iniciativas legislativas se llevaron a cabo con una vocación de implementación completa del 
mainstreaming en todas las áreas y en todas las políticas. Se crearon al mismo tiempo órganos y unidades ex -novo como la Secretaría General de Igualdad, la Comisión Interdepartamental de la Igualdad, los Consejos Participativos o la Unidad Mujer y Ciencia, entre otros. Ahora bien, estos cambios importantes deben comprenderse, no solo como fruto de la voluntad política del momento sino también como resultado del proceso de consolidación de la estrategia estructural del mainstreaming iniciada a mediados de los años noventa.

Con todo, a partir del 2009, con el regreso del PP al gobierno autonómico y bajo un contexto de persistente y profunda crisis económico-financiera se han ido desdibujando sustancialmente los logros obtenidos hasta el momento. Por una parte, se han reducido sustantivamente los recursos dedicados a las políticas de género y por otra, se ha redefinido parte de los contenidos de la agenda de igualdad centrados en la actualidad mayormente en temas relacionados con la conciliación, la violencia de género y el empleo. Entre las transformaciones importantes que afectan al contenido y al alcance las políticas de género se encuentran la supresión del SGI, estructura consolidada que integraba el núcleo de las políticas de igualdad y que constituía un organismo autónomo referente del feminismo institucional en Galicia. Asimismo, la aprobación de la Ley 5/2010, del 23 de junio, por la que se establece y regula una red de apoyo a la mujer embarazada muestra claro un cambio de agenda política.

Por lo tanto, el desmantelamiento progresivo de estructuras de igualdad amparada en el argumento y en el contexto de austeridad y eficiencia en tiempos de crisis económica, avanzó paralelo a un giro estratégico en los contenidos y en la elaboración de la agenda política de género hacia un proyecto con vocación no feminista. Estos nuevos contenidos políticos de género abren el debate acerca de si existe o no una definición homogénea respecto de lo que debe entenderse por igualdad entre mujeres y hombres.

En definitiva, la crisis económica y la deriva conservadora de las políticas de igualdad son los elementos que nos hacen valorar el posible proceso de reversibilidad o desinstitucionalización de la igualdad en Galicia. De acuerdo con Drude Dahlerup (Dahlerup, 2013), la consolidación vigente de las políticas de igualdad de género en las democracias occidentales se encuentra sujeta a lo que la autora denomina "irreversibilidad condicionada", esto es, que los logros conseguidos podrán mantenerse excepto que se produzcan cambios profundos en el contexto. ¿Nos encontramos en ese contexto apto para la reversibilidad de los avances en igualdad?

Tal vez sea desmesurado identificar el reciente recorrido de la igualdad de género en Galicia como un proceso de desinstitucionalización o reversibilidad en un 
sentido amplio paralelo al desmantelamiento creciente del estado de bienestar, no obstante, se han producido importantes pasos atrás, y por ello habrá que continuar pues examinando las medidas y transformaciones que puedan producirse a corto o medio plazo y si cambian de dirección.

\section{BIBLIOGRAFÍA}

ALONSO, ALBA (2007). El mainstreaming y la europeización de las políticas de género en Galicia. Trabajo de investigación tutorado presentado en el Programa de Doctorado Procesos Políticos Contemporáneos. Curso 20062007.

ALONSO, ALBA (2008a). Género y Ciencia. Una visión desde las políticas públicas gallegas. Andaina. Revista del movimiento feminista gallego, $\mathrm{n}^{\circ} 49,47-50$

ALONSO, ALBA (2008b), La perspectiva de género en las políticas científicas: estudio de cuatro Comunidades Autónomas, International Congress New and Old Questions of Women and Gender Research, CIFEX, 14 de mayo, Santiago de Compostela.

ALONSO, ALBA (2014) (en prensa). El mainstreaming de género en España. Valencia: Tirant lo Blanch.

ANDERSON, JAMES (2003). Public Policy-Making: An Introduction. Boston: Houghton Mifflin

ARÁNZAZU, ARANTXA (2003). Las nuevas leyes autonómicas de igualdad de mujeres y hombres. Aequalitas, ${ }^{\circ} 13$ julio-diciembre, 63-74

ARRANZ, FÁTIMA (2000).Algunas notas tecnológicas sobre los Planes de Igualdad de Oportunidades en el marco de las políticas públicas dirigidas a mujeres: el caso español en Arranz, F(Ed.) Las políticas públicas a favor de las mujeres, Madrid: Instituto estudios feministas UCM.

BOOTH, CHRISTINE y BENNET, CINNAMON (2002). Gender Mainstreaming in the European Union. Towards a New Conception and Practice of Equal Opportunities? European Journal of Women's Studies, 9(4), 430-446.

BUSTELO, MARÍA (2004). La evaluación de las políticas de igualdad de género. Madrid: Catarata

BUSTELO, MARÍA y ORTBALS, CANDICE (2007). The evolution of Spanish State Feminism: a Fragmented Landscape en Outshoorn, J y Kantola, J (Eds), Changing State Feminism. Londres: Palgrave Macmillan.

DAHLERUP, DRUDE y MONIQUE LEYENAAR (2013). Breaking Male Dominance in Old Democracies. Londres: Oxford University Press.

DIZ, ISABEL y MARTA LOIS (2011) La institucionalización de la igualdad de género en Galicia: un camino abierto en Revista Gestión y Análisis de Políticas Públicas $\mathrm{n}^{\circ}$ 5, 145-164. 
ESPING-ANDERSEN,GÖSTA (1990). The Three Worlds of Welfare Capitalism. Princeton: Princeton University Press.

KRIZSAN et all (eds)(2012). Institutionalising Intersectionality: The Changing Natures of European Equality Regimes. Basingstoke: Palgrave McMillan.

MAHOU, XOSÉ y BOUZAS, RAMÓN(2007). Gobierno electrónico y organismos de igualdad: examen de los canales de acceso en Diz, I y Lois, M (Eds) Mujeres, instituciones y política. Barcelona: Ediciones Bellaterra.

ORTBALS, CANDICE (2008), Subnational Politics in Spain: New Avenues for Feminist Policymaking and Activism, Politics and Gender, $\mathrm{n}^{\circ}$ 4, 93-116.

RUIZ JIMÉNEZ, ANTONIA (2002) Mecanismos de cambio ideológico e introducción de políticas de género en partidos conservadores: él caso de AP-PP en España en perspectiva comparada. Madrid: Centro de Estudios Avanzados en Ciencias Sociales.

THRELFALL, MÓNICA (1996), Feminist Politics and Social Change in Spain en Threlfall, M. Mapping the Women's Movement, Feminist Politics and Social Transformation in the North, Londres: verso, 111-151. 